Claremont Colleges

Scholarship@ Claremont

All HMC Faculty Publications and Research

HMC Faculty Scholarship

1-1-1995

\title{
Optical Measurements of the Core Radius of High- $\Delta$ Fibers with 1-nm Resolution
}

Peter N. Saeta

Harvey Mudd College

\section{Recommended Citation}

Peter N. Saeta, "Optical measurements of the core radius of high- $\Delta$ fibers with 1-nm resolution," Appl. Opt. 34, 177-182 (1995) doi: 10.1364/AO.34.000177

This Article is brought to you for free and open access by the HMC Faculty Scholarship at Scholarship @ Claremont. It has been accepted for inclusion in All HMC Faculty Publications and Research by an authorized administrator of Scholarship @ Claremont. For more information, please contact scholarship@cuc.claremont.edu. 


\title{
Optical measurements of the core radius of high- $\Delta$ fibers with 1-nm resolution
}

\author{
Peter N. Saeta
}

\begin{abstract}
An optical technique for measuring the core radius of high- $\Delta$ optical fibers is described. Variations in the core radius of step-index fibers can be measured down to a scale of $1 \mathrm{~nm}$.

Key words: Index profile, angular scattering, precision measurements.
\end{abstract}

\section{Introduction}

Step-index single-mode optical fibers with small core diameters and large relative index differences, $\Delta$, suffer from abnormally high loss. The loss is proportional to the inverse fourth power of the wavelength ${ }^{1,2}$ and hence can be described by a Rayleigh scattering coefficient. This coefficient is an increasing function of $\Delta,{ }^{3}$ which rises sharply for $\Delta \geq 0.02 .^{2}$

Although this phenomenon has been recognized for many years, the origin of the excess loss remains unknown. Recent angular measurements show, however, that the light is scattered primarily in the forward direction. ${ }^{4}$ These results agree qualitatively with a theory of scattering loss that arises from random core-radius fluctuations in step-index fibers. 5,6 This theory predicts that radius fluctuations that have an amplitude of only $1 \mathrm{~nm}$ can cause scattering losses approaching $100 \mathrm{~dB} / \mathrm{km},{ }^{6}$ and that fluctuations of quite long spatial periods $(10-100 \mu \mathrm{m})$ can contribute to strong scattering. Such fluctuations might arise from a thermomechanical instability during the draw at the interface between the core and cladding glasses, which, of necessity, havequitedifferent compositions in a high- $\Delta$ fiber. Direct confirmation of this loss mechanism would require sensitive measurements of the core radius with high spatial resolution along the fiber axis.

\footnotetext{
When this work was performed the author was with AT\&T Bell Laboratories, 600 Mountain Avenue, Murray Hill, New J ersey 07974; he is now with the National Institute of Standards and Technology/J oint Institute for Laboratory Astrophysics, Campus Box 440, University of Colorado, Boulder, Col orado 80309-0440.

Received 2 March 1994; revised manuscript received 23 May 1994.

0003-6935/95/010177-06\$06.00/0.

(c) 1995 Optical Society of America.
}

Here a technique for making such measurements is described. The method consists of the scattering of a focused laser beam off the core of the fiber and an analysis of the fringes of the scattering pattern away from the forward direction. It is similar to a conventional light-scattering technique ${ }^{7-9}$ used to provide real-time feedback to control the fiber diameter during the draw. In that technique, the backwardscattering pattern of a $\mathrm{He}-\mathrm{Ne}$ laser beam from the fiber is dominated by the high-contrast air-cladding interface. Although the core significantly perturbs the scattering pattern, ${ }^{8}$ the form of the perturbation is not simple, and it is not straightforward to deduce changes in core radius from changes in the observed scattering pattern. Much more direct information about the core can be obtained when the air-cladding interface is removed by immersion of the fiber in index-matching fluid. Analysis of the resulting scattering pattern and, in particular, the position of the regularly spaced minima permits nanometer-scale fluctuations of the core radius to be observed.

\section{Theory}

Light scattering from fibers has been used for many years to monitor the fiber diameter during the draw and to provide feedback to mi nimize diameter fluctuations. 8,9 The theory for scattering plane el ectromagnetic waves off infinite homogeneous dielectric cylinders has been discussed by many authors. ${ }^{10,11}$ It has been extended to concentric homogeneous infinite cylinders ${ }^{12}$ and infinite cylinders of arbitrary radial index profiles in a perturbative treatment. ${ }^{7}$ Computationally it is perhaps most straightforward to model the index profile of the core as a piecewiseconstant function inside radius $b$ and constant with value $\mathrm{n}_{\text {clad }}$ outside this radius (inside the cladding 
layer and index-matching fluid). We assume, therefore, an index profile of the form

$$
n(r)=\left\{\begin{array}{ll}
n_{m} & \text { for } r_{m-1} \leq r<r_{m} \\
n_{\text {clad }} & \text { for } r>r_{M} \equiv b
\end{array},\right.
$$

which consists of a central region inside $r_{0}$ of index $n_{0}$ and $\mathrm{M}$ annuli of constant index surrounded by $\mathrm{a}$ constant-index region, with the understanding that $r_{-1}=0$. Below we quote Marcuse's essential results and specialize the expressions to the choice of a piecewise-constant-index profile for the core.

Following Marcuse and Presby, ${ }^{7}$ we assume that the electric field of the incident wave is aligned with the fiber axis ( $\mathrm{z}$ axis) and express the total field $\mathrm{E}$ as the sum of an incident plane wave $\mathrm{E}_{\mathrm{inc}}$ and a scattered wave $\mathrm{E}_{\mathrm{sc}}$ :

$$
E=E_{i n c}+E_{s c} \text {. }
$$

Insideannulus $m$ the fiel $d$ can be expressed as

$$
\mathrm{E}_{\mathrm{m}}(\mathrm{r})=\epsilon \sum_{\nu=-\infty}^{\infty}\left[\mathrm{A}_{v}{ }^{\mathrm{m}}{ }_{\nu}\left(\mathrm{n}_{\mathrm{m}} \mathrm{kr}\right)+\mathrm{B}_{\nu}{ }^{\mathrm{m}} \mathrm{Y}_{\nu}\left(\mathrm{n}_{\mathrm{m}} \mathrm{kr}\right)\right] \exp (\mathrm{i} \nu \phi),
$$

total field can be expressed in terms of the Hankel functions $\mathrm{H}_{v}^{(1)}(\mathbf{X})=J_{v}(\mathbf{X})+\mathrm{i} \mathrm{Y}_{v}(\mathbf{X})$ and $\mathrm{H}_{v}(\mathbf{2})(\mathbf{X})=J_{v}(\mathbf{X})-$ $\mathrm{iY}_{\nu}(\mathbf{x})$ as

$$
\begin{aligned}
\mathrm{E}_{\mathrm{inc}}= & \epsilon \exp (\mathrm{ikx})=\frac{\epsilon}{2} \sum_{v=-\infty}^{\infty} \mathrm{i}^{v}\left[\mathrm{H}_{v}{ }^{(1)}\left(\mathrm{kn}_{\mathrm{clad}} \mathrm{r}\right)\right. \\
& +\mathrm{H}_{v}{ }^{(2)}\left(\mathrm{kn}_{\mathrm{clad}} \mathrm{r}\right) \exp (\mathrm{i} \nu \phi), \\
\mathrm{E}= & \epsilon \sum_{v=-\infty}^{\infty} \mathrm{C}_{v} \mathrm{H}_{v}{ }^{(1)}\left(\mathrm{kn}_{\mathrm{clad}} \mathrm{r}\right)+\mathrm{D}_{v} \mathrm{H}_{v}{ }^{(2)}\left(\mathrm{kn}_{\mathrm{clad}} \mathrm{r}\right),
\end{aligned}
$$

where an $\exp (-i \omega t)$ time dependence is assumed and the incident wave travels along the $\phi=0$ direction. Then the outgoing wave is proportional to $\mathrm{H}_{v}{ }^{(1)}(\mathbf{x})$, and, from Eqs. (2), (6), and (7), the scattered wave is given by

$$
\mathrm{E}_{\mathrm{sC}}=\epsilon \sum_{\nu=-\infty}^{\infty}\left(\mathrm{C}_{v}-\frac{\mathrm{i}^{\nu}}{2}\right) \mathrm{H}_{v}^{(1)}\left(\mathrm{n}_{\mathrm{clad}} \mathrm{kr}\right) \exp (\mathrm{iv} \phi) .
$$

Applying the boundary conditions at $r=b$ permits the coefficients $C_{v}$ to be expressed in terms of $A_{v}{ }^{M}$ and $\mathrm{B}_{v}{ }^{\mathrm{M}}$, giving

$$
\mathrm{C}_{v}=\frac{\mathrm{n}_{\mathrm{clad}} \mathrm{H}_{v}{ }^{(2)^{\prime}}(\mathrm{P})\left[\mathrm{A}_{v}{ }^{\mathrm{M}} \mathrm{J}_{v}\left(\mathrm{P}_{\mathrm{M}}\right)+\mathrm{B}_{v}{ }^{\mathrm{M}} \mathrm{Y}_{v}\left(\mathrm{p}_{\mathrm{M}}\right)\right]-\mathrm{n}_{\mathrm{M}} \mathrm{H}_{v}{ }^{(2)}(\mathrm{P})\left[\mathrm{A}_{v}{ }^{\mathrm{M} J}{ }_{v}{ }^{\prime}\left(\mathrm{p}_{\mathrm{M}}\right)+\mathrm{B}_{v}{ }^{\mathrm{M}} \mathrm{Y}_{v}{ }^{\prime}\left(\mathrm{p}_{\mathrm{M}}\right)\right]}{\mathrm{n}_{\mathrm{clad}}\left[\mathrm{H}_{v}{ }^{(1)}(\mathrm{P}) \mathrm{H}_{v}{ }^{(2)^{\prime}}(\mathrm{P})-\mathrm{H}_{v}{ }^{(1)^{\prime}}(\mathrm{P}) \mathrm{H}_{v}{ }^{(2)}(\mathrm{P})\right]}
$$

where $v(\mathbf{x})$ and $Y \boldsymbol{v}(\mathbf{x})$ are the Bessel and the Neumann functions of order $\nu$, respectively, $k=2 \pi / \lambda$ is the vacuum wave number of the incident light, $\mathrm{A}_{v}{ }^{\mathrm{m}}$ and $\mathrm{B}_{v}{ }^{\mathrm{m}}$ are coefficients to be determined by application of boundary conditions, and $\epsilon$ will turn out to be the amplitude of $E_{\text {inc. }}$ F or the solution to remain bounded at the origin, we must have $\mathrm{B}_{v}{ }^{0}=0$. At the interface between successive annuli, $\mathrm{E}_{z}$ and $\mathrm{H}_{\phi} \propto$ $\partial \mathrm{E}_{\mathrm{z}} / \partial \mathrm{r}$ are continuous. This leads to the following pair of equations at each boundary $r_{m}$ :
The detector is placed in the far field, where the asymptotic expansion of the Hankel function, ${ }^{13}$

$$
\mathrm{H}_{v}{ }^{(1)}(\mathbf{X}) \sim(2 / \pi \mathbf{X})^{1 / 2} \exp [\mathrm{i}(\mathbf{X}-\pi v / 2-\pi / 4)],
$$

allows one to simplify the expression for the scattered field, giving

$$
\mathrm{E}_{\mathrm{sc}}=-\epsilon\left(\frac{2}{\pi \mathrm{nkr}}\right)^{1 / 2} \exp [\mathrm{i}(\mathrm{nkr}-\pi / 4)] \sum_{\nu=0}^{\infty} \mathrm{c}_{\nu} \cos \nu \phi,
$$

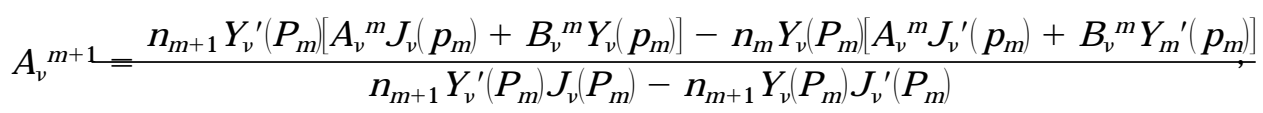

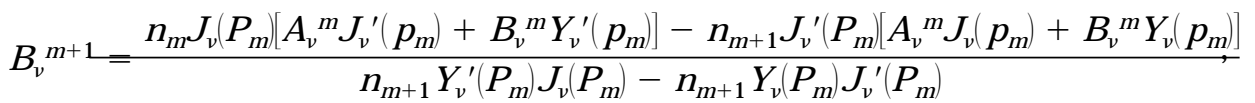

where $p_{m}=n_{m} k r_{m}$ and $P_{m}=n_{m+1} k r_{m}$. Primes represent derivatives with respect to the entire argument of the various functions. These expressions permit the solution for a given angular order $v$ to be propagated from an unknown normalization constant $\mathrm{A}_{v}{ }^{0}$ at $\mathrm{r}=0$ out to the boundary at $\mathrm{r}=\mathrm{b}$, where $\mathrm{A}_{v}{ }^{0}$ can be fixed by continuity with the incoming wave. Outside the core $(r>b)$, the incident field and the where

$$
C_{v}=\epsilon_{v} \exp \left(i \alpha_{v}\right) \cos \alpha_{v},
$$

$\epsilon_{v}=2$ for $v \neq 0$ and $\epsilon_{v}=1$ for $v=0, \mathrm{n}=\mathrm{n}_{\text {clad }}$ in the cladding and the index-matching fluid, $\mathrm{n}=1$ in the 
air outside the cell,

$$
\begin{aligned}
& \tan \alpha_{v}=\frac{\mathrm{n}_{\mathrm{clad}} \mathrm{F}_{v}\left(\mathrm{p}_{\mathrm{M}}\right) \mathrm{Y}_{v}{ }^{\prime}(\mathrm{P})-\mathrm{n}_{\mathrm{M}} \mathrm{F}_{v}{ }^{\prime}\left(\mathrm{p}_{\mathrm{M}}\right) \mathrm{Y}_{v}{ }^{\prime}(\mathrm{P})}{\mathrm{n}_{\mathrm{clad}} \mathrm{F}_{v}\left(\mathrm{p}_{\mathrm{M}}\right) J_{v}{ }^{\prime}(\mathrm{P})-\mathrm{n}_{\mathrm{M}} \mathrm{F}_{v}{ }_{v}\left(\mathrm{p}_{\mathrm{M}}\right) J_{v}{ }_{v}(\mathrm{P})}, \\
& \mathrm{F}_{v}\left(\mathrm{p}_{\mathrm{M}}\right)=\mathrm{A}_{\mathrm{M}} J_{v}\left(\mathrm{p}_{\mathrm{M}}\right)+\mathrm{B}_{\mathrm{M}} \mathrm{Y}_{v}\left(\mathrm{p}_{\mathrm{M}}\right),
\end{aligned}
$$

$\mathrm{p}_{\mathrm{M}}=\mathrm{n}_{\mathrm{M}} \mathrm{kb}$, and $\mathrm{P}=\mathrm{n}_{\mathrm{clad}} \mathrm{kb}$.

Although the sum in Eq. (11) is infinite in the order parameter $v$, the coefficients $C_{v}$ fall off rapidly for $v \gg$ $P$ because the divergence of the Neumann functions sends $\alpha_{v} \rightarrow \pi / 2$. Hence the scattered fiel $d$ is a cosine series with complex coefficients that have approxi-

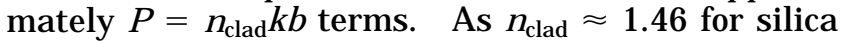
fibers, the number of terms, $\mathrm{P}$, and consequently the spacing of nodes in the scattering pattern, is proportional to the radius $b$, beyond which the index of refraction remains constant, for a given laser wavelength. In the conventional case of scattering from the fiber in air by the use of a He-Nelaser $(\lambda=0.6328$ $\mu \mathrm{m}), \mathrm{P} \approx 620$. This leads to a pattern of very high angular frequency that depends extremely sensitively on the fiber radius and the radial index profile. The portion of this pattern near the backscattered direction has recently been used to measure cladding diameters to $\approx 10 \mathrm{~nm} \cdot{ }^{14}$ In the present case of a nominal step-index fiber with $b \approx 1.2 \mu \mathrm{m}, \mathrm{P}$ is approximately 15 so the fringe spacing is roughly $180^{\circ} / 15=12^{\circ}$.

The discussion thus far has assumed infinite plane waves. In practice, it is necessary to maximize the signal with respect to stray light and to scattering at the cell boundaries and in the index-matching fluid. It is furthermore desirable to focus the light to obtain resolution al ong the fiber axis. A lens was therefore used to focus the laser beam at the fiber core, taking care that the beam waist, $w$, satisfied $w \gg b$. The power detected in an angle $\Delta \phi$ in the far field is then given by

$$
\frac{\mathrm{P}}{\mathrm{P}_{\mathrm{inc}}}=\frac{\lambda \Delta \phi}{2 \pi^{2} \mathrm{~W}}\left|\sum_{\nu=0}^{\infty} \mathrm{c}_{v} \cos \nu \phi\right|^{2},
$$

insofar as the deviations of the incident wave from plane can be ignored. Figure 1 shows the scattered power calculated with Eq. (15) for $\lambda=0.5145 \mu \mathrm{m}, \Delta=$ $2.3 \%$, and $b=1.2 \mu \mathrm{m}$ (heavy curve), assuming $\mathrm{P}_{\text {inc }}=1$ $\mathrm{W}, \Delta \phi=0.25^{\circ}$, and $2 \mathrm{w}=40 \mu \mathrm{m}$. The fine curve shows how the pattern changes for a $1 \%$ increase in $b$. Clearly, if stray light can be suppressed and the pattern around $\phi=90^{\circ}$ can be measured with even modest sensitivity, a resolution of better than $12 \mathrm{~nm}$ can be achieved in these fibers.

\section{Experiment}

The experiments were performed on optical fibers with their polymer jackets removed. The setup was a slight modification of one previously described in detail ${ }^{15}$ and illustrated in Fig. 2. The fibers were held vertically in a chuck with xyz and two-axis tilt adjustment and suspended in a $2.3-\mathrm{cm}$-diameter silica

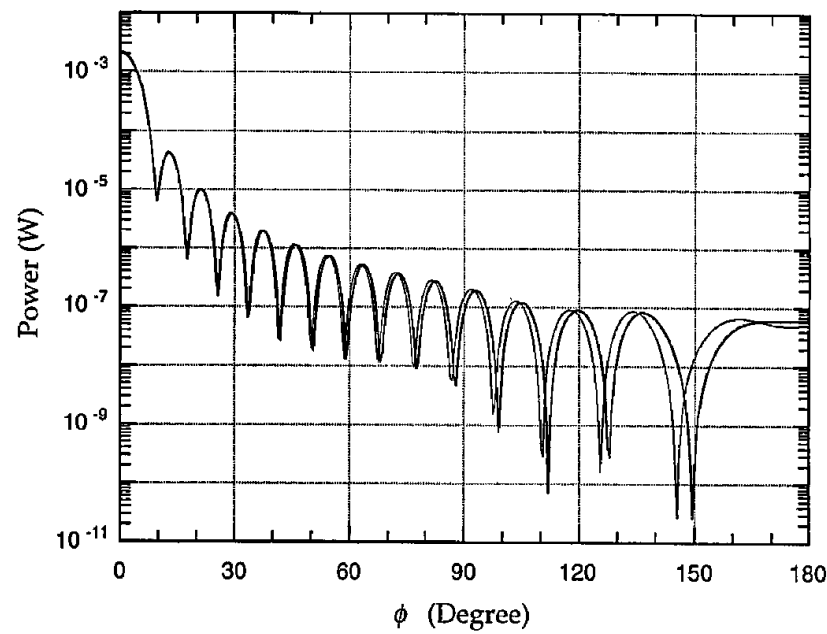

Fig. 1. Calculated scattering pattern for a step-index fiber $(\Delta=0.023, b=1.2 \mu \mathrm{m})$ with $1 \mathrm{~W}$ of $0.5145-\mu \mathrm{m}$ light focused to $2 \mathrm{w}=40 \mu \mathrm{m}$. A slit width of $0.25^{\circ}$ is assumed. The fine curve shows the modified pattern for a $1 \%$ increase in core radius $b$.

cell filled with index-matching fluid. Either the 0.488 - or the $0.5145-\mu \mathrm{m}$ beam of an $\mathrm{Ar}^{+}$laser was focused by a 13.5 -cm focal-length (spherical) lens into the center of the scattering cell, and the transmitted beam in the forward direction was dumped into a calorimeter to minimize stray light. The beam was vertically polarized (parallel to the fiber axis) and chopped to permit lock-in detection. Typical beam power was $1 \mathrm{~W}$. The scattered light was detected with an unamplified Si photodiode mounted upon an optical rail atop a computer-controlled rotation stage at a distance of $\sim 20 \mathrm{~cm}$ from the fiber. The acceptance angle of the detector was determined by an

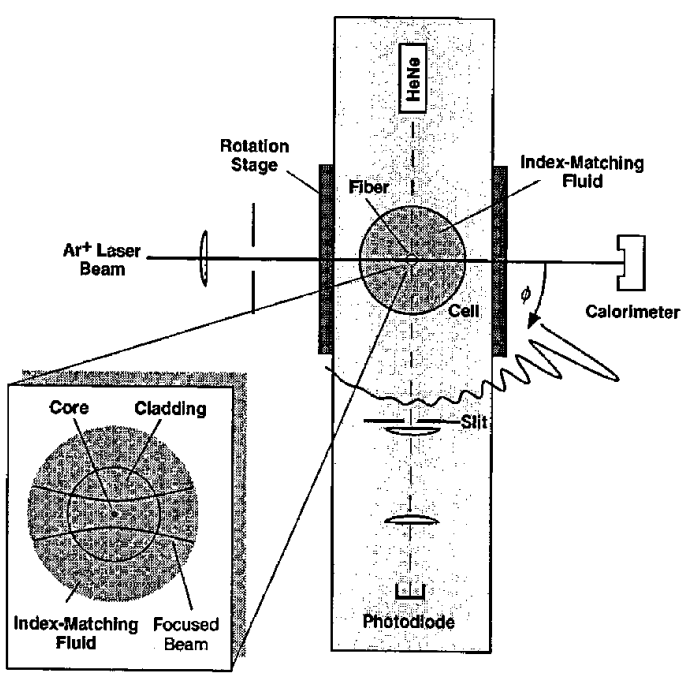

Fig. 2. Experimental setup. The polarization of the incident $\mathrm{Ar}^{+}$ laser beam is out of the plane of the figure, parallel to the fiber axis. The incident beam is focused at the fiber core as illustrated in the expanded view at lower left. The scattered light lies in the plane of the figure, peaked strongly in the forward direction, as shown schematically in the figure. The He-Ne laser is used for alignment; it is switched off during measurements. 


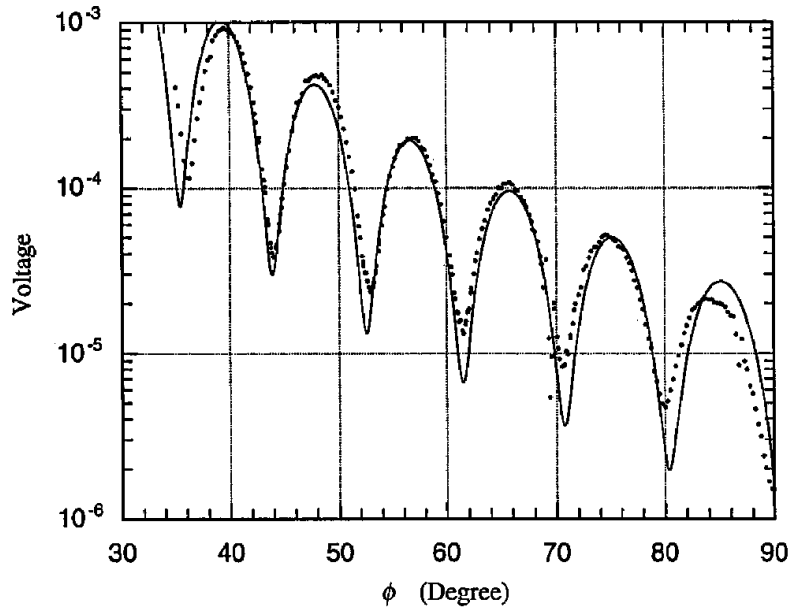

Fig. 3. Observed scattering pattern for fiber A, a nominally step-index high- $\Delta$ fiber. The solid curve is the best fit to the bi-Fermi functional form (see Table 1), giving a core diameter of $2.44 \mu \mathrm{m}$.

adjustable-width vertical slit mounted $10 \mathrm{~cm}$ from the fiber on the rotating rail; typical acceptance angles were $0.1^{\circ}-0.3^{\circ}$. To facilitate alignment, a $\mathrm{He}-\mathrm{Ne}$ laser was mounted on the rail opposite the detector and positioned so that its beam passed through the center of rotation of the stage and was centered on the detector. Care was taken to center both the cell and the fiber about the rotation axis of the stage and to adjust the tilt of the fiber to produce a horizontal scattering pattern.

\section{Results and Discussion}

The high- $\Delta$ fiber used for most of the scattering experiments (fiber A) had a silica cladding layer and a germanosilicate core $\left(18 \% \mathrm{GeO}_{2}\right)$, yiel ding a nominal $\Delta \mathrm{n}$ of $0.030(\Delta=2.1 \%)$ and core radius of $1.2 \mu \mathrm{m}$. When the fiber was immersed in index-matching fluid and illuminated with either $\mathrm{Ar}^{+}$line, the scattering pattern was easily visible by eye through angles exceeding $90^{\circ}$ from the forward direction and was symmetric about the forward direction. The intensity of the scattered light dropped rapidly with increasing azimuthal angle $\phi$ and exhibited a sequence of roughly equally spaced minima, as expected from the theory. When the index-matching fluid and the cladding had slightly different indices of refraction, the pattern was modulated by a shallow high-frequency interference pattern with a line spacing of a fraction of a degree. The absence of such a high-frequency pattern confirmed the proper choice of matching fluid. Further proof that the core was the scattering object came from the translation of the fiber through the beam waist, in which case strong scattering was observed over a range roughly equal to the beam waist and appreciably less than the $125-\mu \mathrm{m}$ diameter of the fiber.

The measured scattering pattern from fiber $A$ is shown in Fig. 3 for the range $35^{\circ} \leq \phi \leq 90^{\circ}$. On the vertical scale of diode voltage, the background level detected when the fiber was removed from the cell was between $10^{-7}$ and $10^{-6} \mathrm{~V}$ over this range. The data agree qualitatively with the cal culated pattern of

Table 1. Index Profile Functions Used to Fit Scattering Data ${ }^{a}$

\begin{tabular}{|c|c|c|c|}
\hline Step index & $n(r)=\{$ & $\begin{array}{ll}n_{\text {core }} & r<b \\
n_{\text {clad }} & r>b\end{array}$ & \\
\hline Smoothed step & $n(r)=\{$ & $\begin{array}{l}n_{\text {core }} \\
n_{\text {clad }}+\Delta n(a-r) / 2 w \\
n_{\text {clat }}\end{array}$ & $\begin{array}{l}r<a-w \\
a-w<r<a+w \\
r>a+w=b\end{array}$ \\
\hline Exponential & $n(r)=\{$ & $\left\{\begin{array}{l}n_{\text {core }} \\
n_{\text {clad }}+\Delta n \exp \left(-\frac{r-a}{w}\right)\end{array}\right.$ & $\begin{array}{l}r<a \\
r>a\end{array}$ \\
\hline Fermi & $n(r)=n$ & $a_{\text {clad }}+\frac{\Delta n}{1+\exp \left(\frac{r-a}{w}\right)}$ & \\
\hline Bi-Fermi & $n(r)=$ & $\begin{array}{l}n_{\text {core }}-\frac{\Delta n}{1+\exp \left(\frac{a-r}{w_{1}}\right)} \\
n_{\text {clad }}+\frac{\Delta n}{}\end{array}$ & $r<a$ \\
\hline
\end{tabular}

al $\mathrm{n}$ these expressions, $\Delta \mathrm{n}=\mathrm{n}_{\text {core }}-\mathrm{n}_{\text {clad, }}$ b is a radius beyond which $\mathrm{n}=\mathrm{n}_{\text {clad, }}$, and $\mathrm{a}$ is the radius that satisfies $\mathrm{n}(\mathrm{a})=1 / 2\left(\mathrm{n}_{\text {core }}+\mathrm{n}_{\text {clad }}\right)$. 
Fig. 1, having roughly the same fringe spacing, although the successive maxima fall off more rapidly than in the calculation for the idealized step-index core profile. Ideally, one would like to deduce the index profile directly from the measured scattering pattern. Unfortunately, this inversion problem is numerically unstable. Instead, an attempt was made to fit the data by the use of a core index-profile function with a smoothed transition from $n_{\text {core }}$ to $n_{\text {clad. }}$. Several forms were tried, as shown in Table 1 . The curve in Fig. 3 shows the best fit obtained, which used the bi-Fermi functional form. The fit follows both the spacing of the minima and the decrease in the maxima with $\phi$ fairly well; the discrepancy at the minima results from the finite slit width.

The index profile that corresponds to the fit of Fig. 3 is compared in Fig. 4 with measurements made by the use of the refracted near-field method with a York Model S14 fiber profiler. The profile obtained from the fit has an approximate transition width of $0.1 \mu \mathrm{m}$, whereas the measured one is roughly eight times broader. The broadening is primarily due to the $\sim 0.5-\mu \mathrm{m}$ spot size of the laser used to make the measurements, which necessarily broadens abrupt index changes. The scattering pattern calculated from the measured profile shown in Fig. 4 falls off much more rapidly with $\phi$ than the data and, unlike the data, shows minima of quite variable depths. It is likely, therefore, that the fit gives a much more accurate picture of the index profile.

Simulations with the functions of Table 1 showed that the pattern is much more sensitive to the core radius, $b$, than to $\Delta$. They also displayed the common features that, for small changes in $b$,

$$
\frac{\delta b}{\mathrm{~b}} \propto-\frac{\delta \phi_{\min }}{\phi_{\min }},
$$

where $\phi_{\min }$ is the position of a local minimum in the

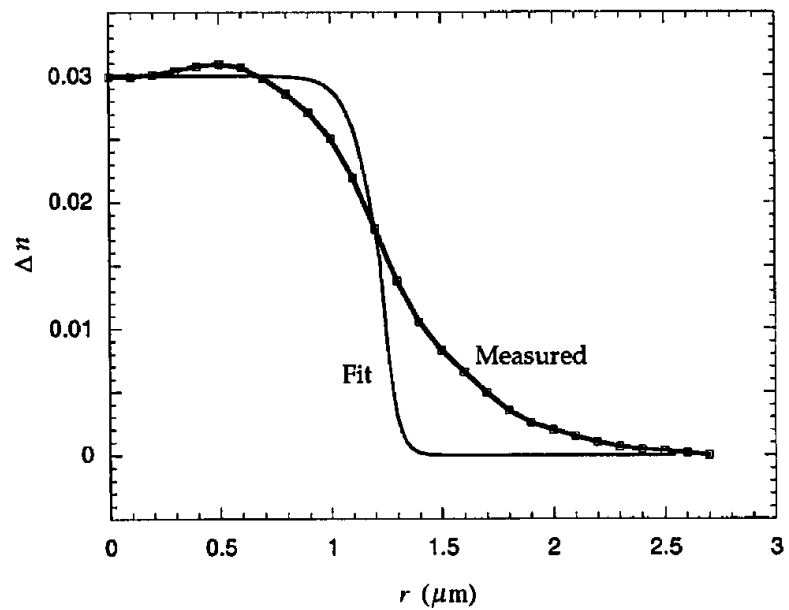

Fig. 4. Index profile of fiber A as measured with the refracted near-field method with a York Model S14 fiber profiler (heavy curve) and as obtained from the fit shown in Fig. 3 (thin curve). The difference illustrates the broadening introduced by the $\sim 0.5-\mu \mathrm{m}$ focal spot of the profiler.

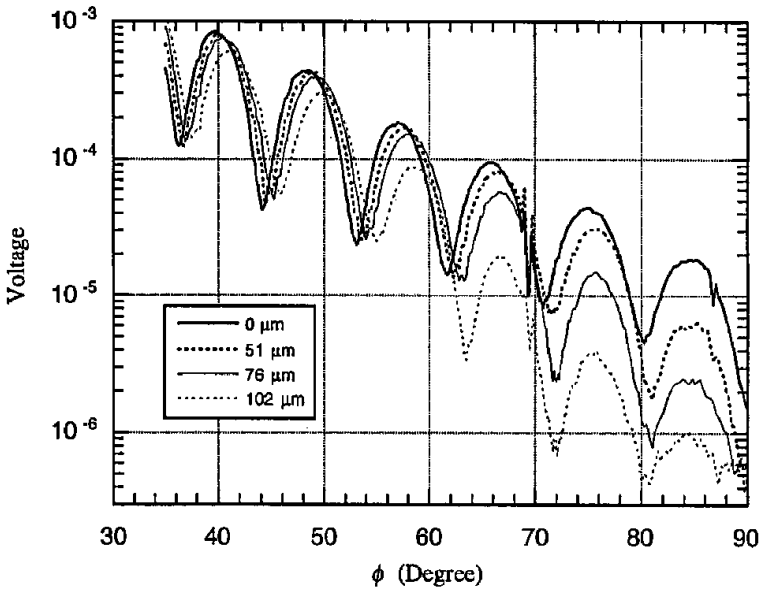

Fig. 5. Four scattering patterns obtained from the neck-down region of a tip made from fiber $A$. The trace labeled $0 \mu \mathrm{m}$ corresponds to a position just outside the heated region and matches the pattern of the unmodified fiber. With an increase in coordinate $z$ along the fiber, the minima shift to larger $\phi$, as expected for a decreasing core radius.

scattering pattern. This suggests that even if a satisfactory fit cannot be made to the data, useful information about changes in the core radius can be obtained by the accurate measurement of the position of one or more minima in the pattern as a function of distance $z$ al ong the fiber.

To investigate the sensitivity of this information, a segment of fiber A was laser heated under tension, which caused it to neck down to a point and break in the heated region. Scattering measurements were then made of this tip, starting in the region of the fiber that had not been heated. Four of these measurements are shown in Fig. 5. As expected, the minima shift to larger values of $\phi$ as the core radius decreases from the unmodified portion of the fiber $(z=0 \mu \mathrm{m})$ into the neck-down region $(z>0)$. With expression (16), the relative change in radius, $\delta b / b$, was calculated based on the minima near $44^{\circ}$ and $53^{\circ}$,

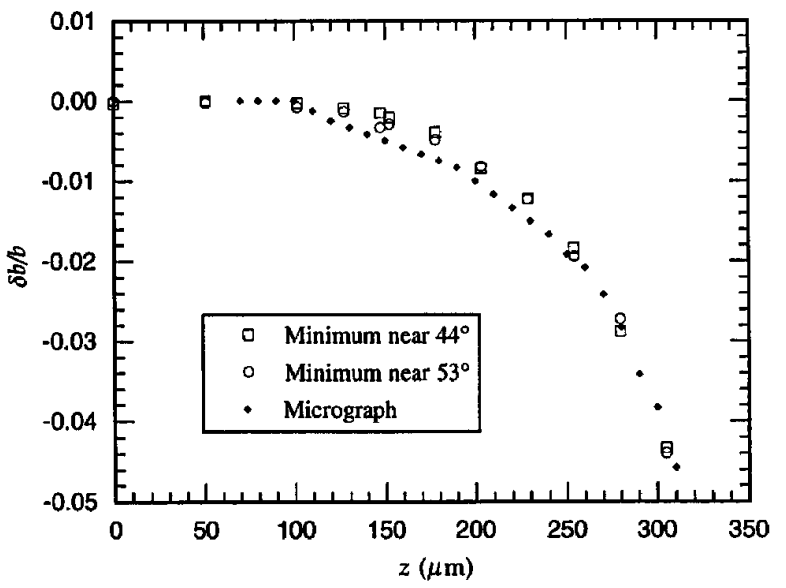

Fig. 6. Plot of the relative change in core radius along the length of the fiber in the neck-down region. The filled diamonds were taken from a digitized micrograph; the open symbols were obtained from the relative shift in the position of minima near $44^{\circ}$ and $53^{\circ}$. 
and the results are shown as a function of $z$ in Fig. 6 . These are compared with a profile deduced from a micrograph taken of the neck-down region of the fiber, based on the assumption that the relative change of the core is equal to the relative change of the outside fiber diameter. The agreement between the two curves calculated from the shift in minima is excellent, better than a part in 1000 in the unmodified region $(z<100 \mu \mathrm{m})$ and quite good throughout. As b $\approx 1.2 \mu \mathrm{m}$, this implies that the technique can resolve fluctuations in a core radius smaller than $1 \mathrm{~nm}$.

\section{Conclusions}

Theangular scattering pattern produced from a high- $\Delta$ nominally step-index fiber immersed in index-matching fluid has been measured and shown to provide a sensitive measure of the core radius. Although attempts to deduce the exact index profile from the scattering pattern were unsuccessful, small variations in core radius produce proportional shifts in the angular position of sharp minima in the scattering pattern. These shifts were used to profile the neckdown region of a fiber tip with a resolution of better than $1 \mathrm{~nm}$ in the core radius.

I thank W. A. Reed and L. R. Copeland for help in setting up the experiment, B. I. Greene, D. M. Krol, and S. L. McCall for stimulating discussions, D. J . DiGiovanni for the fiber samples, R. E. Betzig for preparing the fiber tips, and C. R. Kurkjian for micrographs of the tips.

\section{References}

1. B. J . Ainslie, K.J . Beales, C. R. Day, and J . D. Rush, "Interplay of design parameters and fabrication conditions on the performance of monomode fibres made by MCVD," IEEE J . Quantum Electron. QE-17, 854-857(1981).
2. S. T. Davey, D. L. Williams, D. M. Spirit, and B. J . Ainslie, "The fabrication of low loss high NA silica fibres for Raman amplification," in Fiber Laser Sources and Amplifiers, M. J . Digonnet, ed., Proc. Soc. Photo-Opt. Instrum. Eng. 1171, 181-191 (1989).

3. T. Tomaru, M. Kawachi, M. Yasu, T. Miya, and T. Edahiro, "VAD single-mode fibres with high $\Delta \mathrm{n}$ values," Electron. Lett. 17, 731-732 (1981).

4. L. Copeland, AT\&T Bell Laboratories, 600 Mountain Avenue, Murray Hill, N.J . 07090 (personal communication, 1993).

5. D. Marcuse and R. M. Derosier, "Mode conversion caused by diameter changes of a round dielectric waveguide," Bell Sys. Tech. J . 48, 3217-3232 (1969).

6. D. Marcuse, "Microdeformation losses of single-mode fibers," Appl. Opt. 23, 1082-1091 (1984).

7. D. Marcuse and H. M. Presby, "Light scattering from optical fibers with arbitrary refractive-index distributions," J . Opt. Soc. Am. 65, 367-375 (1975).

8. H. M. Presby, "Refractive index and diameter measurements of unclad optical fibers,"J . Opt. Soc. Am. 64, 280-284 (1974).

9. H. M. Presby and D. Marcuse, "Refractive index and diameter determinations of step index optical fibers and preforms," Appl. Opt. 13, 2882-2885 (1974).

10. J. A. Stratton, Electromagnetic Theory (McGraw-Hill, New York, 1941), Chap. 6, pp. 349-391.

11. H. C. van de Hulst, Light Scattering by Small Particles (Dover, New York, 1981), Chap. 15, pp. 300-301.

12. M. Kerker and E. Matijevic, "Scattering of electromagnetic waves from concentric infinite cylinders," J . Opt. Soc. Am. 51, 506-508 (1961).

13. M. Abramowitz and I. A. Stegun, eds., Handbook of Mathematical Functions (Dover, New York, 1972), Chap. 9, pp. 355-389.

14. M. B. van der Mark and L. Bosselaar, "Noncontact calibration of optical fiber cladding diameter using exact scattering theory," J. Lightwave Technol. 12, 1-5(1994).

15. A. E. Neeves and W. A. Reed, "Experimental apparatus for measurement of the angular, polarization, and wavelength dependence of light scattering from the visible to the infrared in bulk glass samples," Appl. Opt. 31, 2072-2077 (1992). 